\title{
Turbulent motions and shocks waves in galaxy clusters simulated with adaptive mesh refinement
}

\author{
F. Vazza ${ }^{1,2}$, G. Brunetti ${ }^{2}$, A. Kritsuk ${ }^{3}$, R. Wagner ${ }^{3,4}$, C. Gheller ${ }^{5}$, and M. Norman ${ }^{3,4}$ \\ 1 Dipartimento di Astronomia, Universitá di Bologna, via Ranzani 1, 40127 Bologna, Italy \\ e-mail: vazza@ira.inaf.it \\ 2 INAF/Istituto di Radioastronomia, via Gobetti 101, 40129 Bologna, Italy \\ 3 Ctr. for Astrophysics and Space Sciences, U.C. San Diego, La Jolla, CA 92093, USA \\ 4 Physics Department, U.C. San Diego, La Jolla, CA 92093, USA \\ 5 CINECA, High Performance System Division, Casalecchio di Reno-Bologna, Italy
}

Received 19 May 2009 / Accepted 30 June 2009

\section{ABSTRACT}

\begin{abstract}
We have implemented an adaptive mesh refinement criterion explicitly designed to increase spatial resolution around discontinuities in the velocity field in ENZO cosmological simulations. With this technique, shocks and turbulent eddies developed during the hierarchical assembly of galaxy clusters are followed with unprecedented spatial resolution, even at large distances from the clusters center. By measuring the spectral properties of the gas velocity field, its time evolution and the properties of shocks for a reference galaxy cluster, we investigate the connection between accretion processes and the onset of chaotic motions in the simulated intergalactic medium over a wide range of scales.
\end{abstract}

Key words. galaxies: clusters: general - methods: numerical - intergalactic medium - large-scale structure of Universe

\section{Introduction}

The intergalactic medium (IGM) in galaxy clusters is likely turbulent, at some level: this is claimed from several independent theoretical and numerical approaches (e.g. Bryan \& Norman 1998; Ricker \& Sarazin 2001; Brunetti et al. 2001; Inogamov \& Sunyaev 2003; Dolag et al. 2005; Subramanian et al. 2006; Vazza et al. 2006; Brunetti \& Lazarian 2007; Nagai et al. 2007; Iapichino \& Niemeyer 2008). A number of observational evidences has also been published in the last few years. Using a mosaic of XMM-Newton observations of the Coma cluster, Schuecker et al. (2004) obtained spatially-resolved gas pressure maps which indicate the presence of a significant amount of turbulence, with a spectrum of the fluctuations consistent with a Kolmogorov turbulence. Additional evidences of turbulent motions inside nearby galaxy clusters came from the observation of pseudo-pressure fluctuations in Abell 754 using XMM (Henry et al. 2004) and from the non detection of resonant scattering in the Perseus cluster (Churazov et al. 2004). Also studies of Faraday rotation allow a complementary approach and suggest that the IGM magnetic field is turbulent on a broad range of scales (Murgia et al. 2004; Govoni et al. 2006; Ensslin \& Vogt (2006).

Detailed X-ray analysis performed in nearby cool-core galaxy clusters (e.g. Fabian et al. 2003; Churazov et al. 2004; Graham et al. 2006; Ota et al. 2006) suggest that the turbulent velocity field is subsonic at the scale of the cluster cores. Also, limits to the amount of turbulence in the IGM were recently derived by Churazov et al. (2008), suggesting that the amount of non-thermal pressure within $\sim 50 \mathrm{kpc}$ from the central galaxies in Perseus and Virgo clusters cannot exceed $\sim 10-20$ per cent of the thermal energy budget at the same radius.
In addition, the phenomenology of diffuse radio halo emission suggests a scenario in which turbulent MHD modes, excited during cluster mergers, may re-accelerate the relativistic emitting particles (e.g. Ferrari et al. 2008; Brunetti et al. 2008; Cassano 2009, and references therein). Remarkably, the interplay between cosmic rays (CR) and turbulent magnetic fields may drive still poorly explored plasma processes that may potentially affect our simplified view of the IGM (Subramanian et al. 2006; Schekochihin et al. 2009; Brunetti \& Lazarian 2007; Guo \& Oh 2008). From the theoretical point of view, turbulence can be injected in the IGM by several mechanisms: plasma instabilities, cluster mergers and shock waves, wakes of galaxies moving into the IGM, outflows from AGNs hosted in the center of galaxy clusters and galactic winds. The total energy budget in form of turbulent motions inside galaxy clusters, as well as their distribution and their connection with cluster dynamics and non gravitational process in galaxy clusters are sill open fields of study and cosmological numerical simulations are potentially able to provide a great insight in the characterization of the above phenomena.

Early Eulerian numerical simulations of merging clusters (e.g., Bryan \& Norman 1998; Ricker \& Sarazin 2001) provided the first reliable representations of the way in which turbulence is injected into the IGM by merger events. More recently, high resolution Lagrangian (Dolag et al. 2005; Vazza et al. 2006) and Eulerian simulations (Nagai et al. 2007; Iapichino \& Niemeyer 2008) found that a sizable amount of pressure support (i.e. 10 percent of the total pressure inside $0.5 R_{\text {vir }}$ ) in the IGM is sustained by chaotic motions. Also, the amount of turbulent energy delivered by mergers and accretions is found to scale with the thermal energy of simulated galaxy clusters (Vazza et al. 2006). 
Table 1. Main characteristics of the runs. "D" stands for AMR triggered by gas/DM over-density, while "V" stands for AMR triggered by velocity jumps. $\Delta$ is the peak gas spatial resolution. $\delta$ specifies the value adopted to trigger AMR, see Sect. 3 for explanations.

\begin{tabular}{c|c|c|c|c}
\hline \hline ID & $N_{\text {grid }}$ & $M_{\mathrm{dm}}\left[M_{\odot} / h\right]$ & $\Delta[\mathrm{kpc}]$ & AMR \\
\hline v256-4 & $256^{3}$ & $6.76 \times 10^{8}$ & 18 & $\mathrm{D}+\mathrm{V}(\delta>10)$ \\
$\mathrm{v} 256-3$ & $256^{3}$ & $6.76 \times 10^{8}$ & 36 & $\mathrm{D}+\mathrm{V}(\delta>3)$ \\
v128-3 & $128^{3}$ & $5.39 \times 10^{9}$ & 36 & $\mathrm{D}+\mathrm{V}(\delta>3)$ \\
v64-3 & $64^{3}$ & $4.32 \times 10^{10}$ & 36 & $\mathrm{D}+\mathrm{V}(\delta>3)$ \\
d128 & $128^{3}$ & $5.39 \times 10^{9}$ & 36 & $\mathrm{D}$ \\
v128-10 & $128^{3}$ & $5.39 \times 10^{9}$ & 36 & $\mathrm{D}+\mathrm{V}(\delta>10)$ \\
v128-1 & $128^{3}$ & $5.39 \times 10^{9}$ & 36 & $\mathrm{D}+\mathrm{V}(\delta>1)$ \\
v128-z2 & $128^{3}$ & $5.39 \times 10^{9}$ & 36 & $\mathrm{D}+\mathrm{V}(\delta>3, z>2)$ \\
\hline
\end{tabular}

Despite the tremendous capability that Lagrangian simulations have in resolving the smallest structures within galaxy clusters, they may suffer of serious limitations in modeling fluid instabilities, mostly because of the effects played by the artificial viscosity employed to solve hydro equations (e.g. Agertz et al. 2007; Tasker et al. 2008; Mitchell et al. 2009). Therefore, the use of an Eulerian scheme free of artificial viscosity, as the Piecewise Parabolic Method adopted in the ENZO, can provide an important insight in all the above points. On the other hand Eulerian schemes with fixed grid resolution are typically limited by their low spatial resolution so that the application of adaptive mesh refinement (AMR) techniques is mandatory to achieve adequate spatial detail in the simulations.

In this work, we present first results from the application of a new mesh refinement criterion to ENZO simulations, which allows to follow at the same time shocks and turbulent motions with unprecedented resolution up to large distances from the galaxy cluster center. For the simulations presented here, we assume a $\Lambda \mathrm{CDM}$ cosmology with parameters $\Omega_{0}=1.0$, $\Omega_{\mathrm{BM}}=0.0441, \Omega_{\mathrm{DM}}=0.2139, \Omega_{\Lambda}=0.742$, Hubble parameter $h=0.72$ and a normalization of $\sigma_{8}=0.8$ for the primordial density power spectrum.

\section{Numerical code and setup}

ENZO is an AMR cosmological hybrid code highly optimized for supercomputing (Bryan \& Norman 1997, 1998; Norman \& Bryan 1999; Bryan et al. 2001, O'Shea et al. 2004; Norman et al. 2007). It couples an N-body particle-mesh solver with an adaptive mesh method for ideal fluid-dynamics (Berger \& Colella 1989). ENZO adopts an Eulerian hydrodynamical solver based on the the piecewise parabolic method (PPM, Woodward \& Colella 1984), that is a higher order extension of Godunov's shock capturing method (Godunov 1959). The PPM algorithm belongs to a class of schemes in which an accurate representation of flow discontinuities is made possible by building into the numerical method the calculation of the propagation and interaction of non-linear waves. It is at least second-order accurate in space (up to the fourth-order, in the case of smooth flows and small time-steps) and second-order accurate in time. The PPM method describes shocks with high accuracy and has no need of artificial viscosity, leading to an optimal treatment of energy conversion processes, to the minimization of errors due to the finite size of the cells of the grid and to a spatial resolution close to the nominal one. In the cosmological framework, the basic PPM technique has been modified to include the gravitational interaction and the expansion of the Universe.

We present here the simulation of a cubic volume of side $75 \mathrm{Mpc}$ starting from $z=30$, and applying AMR within a

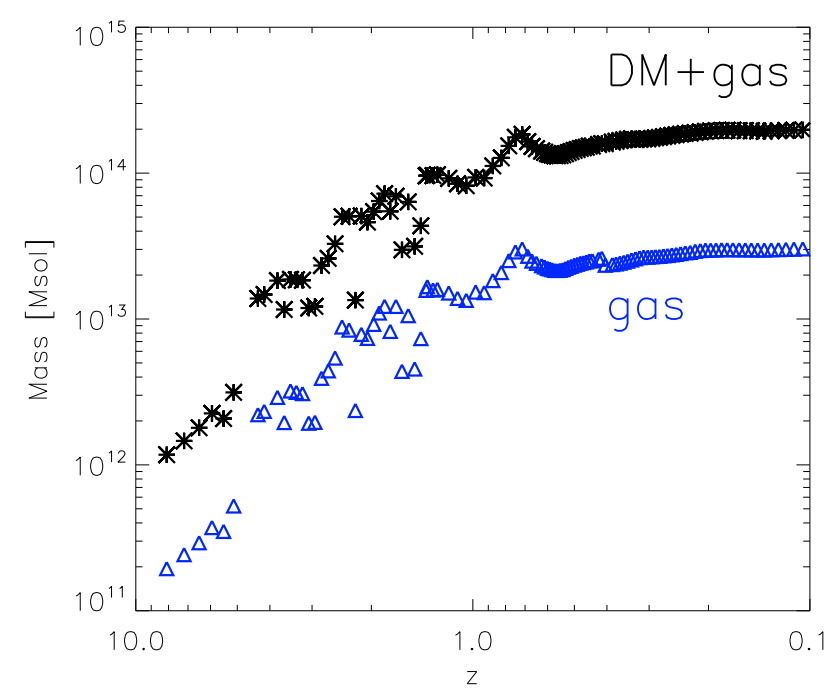

Fig. 1. Redshift evolution of the Dark matter plus gas mass (black), and of total gas mass (blue) inside the virial radius of the galaxy cluster studied in this work.

sub-volume of side $7.5 \mathrm{Mpc}$, centered on a $\sim 2 \times 10^{14} M_{\odot}$ galaxy cluster. We re-simulate this volume under different configurations, as reported in Table 1. The mass resolution of Dark Matter (DM) particles ranges from $6.76 \times 10^{8} M_{\odot}(\mathrm{v} 256-3$ and v2564) to $4.32 \times 10^{10} M_{\odot}(v 64-3)$, corresponding to minimum root grid spatial resolutions from $292 \mathrm{kpc}$ to $1.172 \mathrm{Mpc}$. The maximum spatial resolution in the region where AMR is applied is $\Delta=36 \mathrm{kpc}$ in all the simulations except for the case of $\mathrm{v} 256-4$, where $\Delta=18 \mathrm{kpc}$. All runs are non radiative, and furthermore no treatment of reionization background due to AGN and or massive stars is modeled here.

In all the above simulations, the galaxy cluster is formed through a major merger at $0.8<z<1$, and visual inspection shows that its perturbed dynamical state stays till later epochs, due to further accretions. Figure 1 shows the redshift evolution of the total mass and of the gas mass inside the virial cluster region, measured according to the spherical over-density centered on the density peak where the galaxy cluster forms.

Computations described in this work were performed using the ENZO code developed by the Laboratory for Computational Astrophysics at the University of California in San Diego (http://lca.ucsd.edu).

\section{Adaptive mesh refinement technique for turbulent motions}

The first application of AMR to the study of turbulence in the inter stellar medium was reported in Kritsuk et al. (2006). Iapichino \& Niemeyer (2008) applied a refinement criterion based on the gas velocity field (analyzing curl and divergence of velocity), in order to study turbulence in cosmological ENZO simulations. Motivated by the above results, here we report on an exploratory study where a grid refinement scheme based on the analysis of one dimensional jumps in the velocity field is introduced in ENZO. In order to apply this method at full power to ENZO simulations we combine the implementation of the standard grid refinement criterion, customary adopted in cosmological simulations (based on over density), with a new grid refinement criterion based on the analysis of the jump of velocity, 
$\Delta v$, across cells. This choice ensures that shocks can be studied with the highest available resolution in simulated galaxy clusters, contrary to usual AMR runs, and at the same time this refinement scheme allows to increase the spatial resolution around turbulent features in the simulated galaxy clusters.

In Teyssier (2002) 1-D tests are presented to assess the importance of refining shocks (according to a pressure criterion) in cosmological simulations. The major findings were that: a) relevant numerical instabilities do occur when simulated shocks move from a low resolution to a high resolution region (e.g. from a low density to a high density environment); b) since most of cosmological shocks move from high density (collapsing) regions to low density regions, explicitly refining on shocks can be safely avoided in the run time calculation of expanding accretion shocks (such as those developed in a standard Zeldovich pancake collapse). However, in the high resolution simulation of galaxy cluster we present here, we expect significant departures from any idealized self-similar model of shocks (e.g. Molnar et al. 2009), and the additional refinement on shock waves is a interesting option.

In more detail, we propose to use the normalized $1-\mathrm{D}$ velocity jump across $1-\mathrm{D}$ patches in the simulation, $\delta \equiv\left|\Delta v / v_{\mathrm{m}}\right|$ (where $v_{\mathrm{m}}$ is the minimum velocity, in modulus, over the cells in the patch) to trigger grid refinement. Even if this method is highly simplified respect to that employed in Iapichino \& Niemeyer (2008), in the next sections we will show that it produces a significant step forward, with no significant extra expense of computational effort, in both the study of shock waves and the spectral characterization of the gas velocity field inside galaxy clusters.

In particular we recursively analyze the velocity jumps across three adjacent cells at a given AMR level, and increase the resolution (by a factor 2 in cell size) for the cells of the patch whenever $\delta$ is larger than a threshold value. At the same time, also the standard AMR method triggered by gas/DM overdensity is applied (e.g. Norman et al. 2007); the over-density threshold is set $\delta_{\rho}=\delta \rho / \rho=2$ (where $\rho$ can be either Dark Matter of gas density) for all runs. We notice that this threshold is smaller than what usually taken in similar works (e.g. $\delta_{\rho}=4$ in O'Shea et al. 2004; Nagai et al. 2006; Iapichino \& Niemeyer 2008) and thus typically much more volume is refined in the simulations presented here.

We adopt as reference value $\delta=3$ and allow for a number of AMR levels up to the maximum resolution of $\Delta=36 \mathrm{kpc}$. In one case, we also perform a run using the same setup of the v256-3 run, but allowing for one more AMR level (4 levels instead of 3), reaching the maximum resolution of $18 \mathrm{kpc}$ (v256-4). Finally, we present results for $\delta=10$ (v128-10) and $\delta=1(\mathrm{v} 128-1)$, in order to assess the convergence of our results (Sects. 4.2-4.3).

A reference simulation is also produced where only the gas/DM over-density criterion is used to trigger mesh refinements (d128), along with a test run where the AMR criterion triggered by velocity jumps is added to the standard one only starting from $z \leq 2$ (v128-z2). The latter run is designed in order to establish whether it is feasible to apply the novel mesh refinement criterion starting only at later cosmic epochs, where clusters formation starts, saving some computational effort.

\section{Results}

\subsection{Comparison with standard AMR runs}

Figure 2 shows 2-D slices of gas density and temperature comparing runs v128-3 and $\mathrm{d} 128$ at $z=0.1$. Unlike in the standard

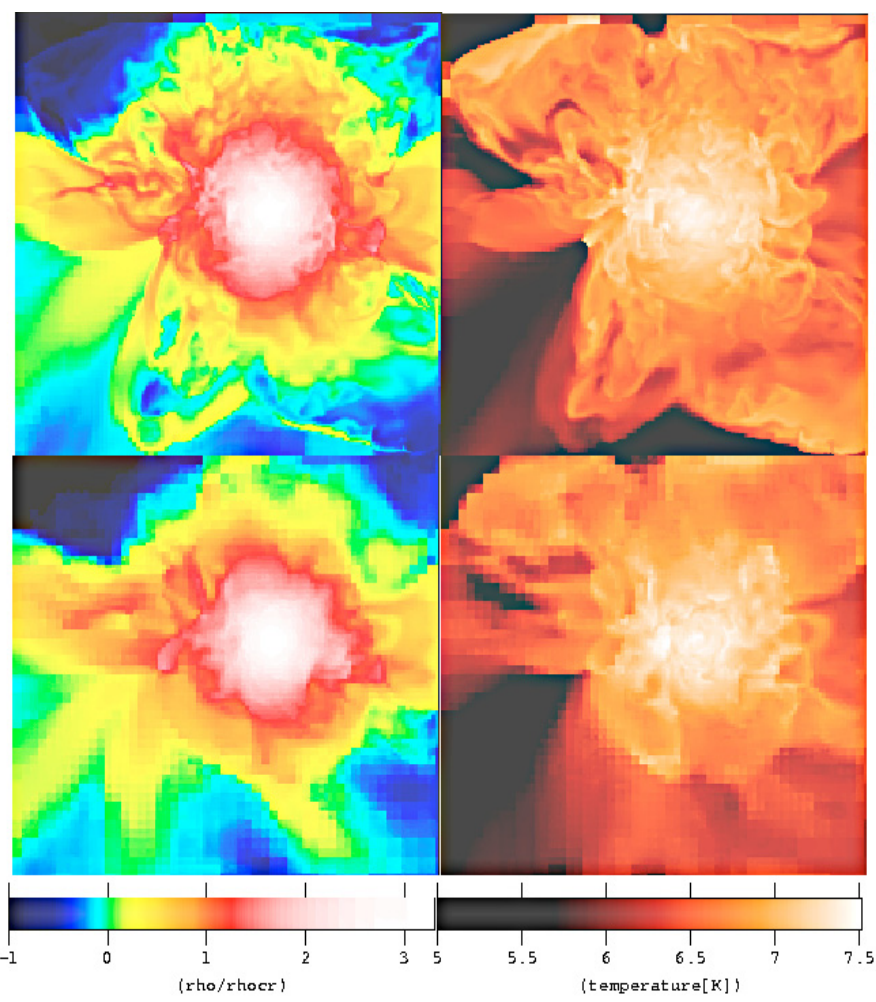

Fig. 2. Gas density and temperature slices for the AMR region of the v128-3 run (upper panels), and of the $\mathrm{d} 128$ run (lower panel). The gas density is normalized to the cosmological critical gas density: rhocr $\equiv \rho_{\mathrm{cr}} \equiv \rho_{\mathrm{cr}, 0} f_{\mathrm{b}}$, where $\rho_{\mathrm{cr}, 0}=3 H(z)^{2} / 8 \pi G$ is the cosmological critical density $\left(H(z)\right.$ is the Hubble constant), and $f_{\mathrm{b}}$ is the cosmic baryon fraction. The side of the image is $7.5 \mathrm{Mpc}$ and the depth along the line of sight is $36 \mathrm{kpc}$.

mesh refinement triggered by gas/DM over-density, with the new AMR criterion shocks and chaotic motions are followed at the highest available resolution in the run, $\Delta=36 \mathrm{kpc}$, up to large $(\sim 3-4 \mathrm{Mpc})$ distances from the cluster center. The difference between the two approaches is remarkable at all stages in the evolution of the cluster, and especially in highlighting strong shock waves excited during the major merger event, as shown in the temperature maps of Fig. 3.

In Fig. 4, we show the distribution of the volume occupied by cells at the different available AMR levels, comparing the results from $v 128-3$ and $\mathrm{d} 128$ at $z=0.1$. This shows that the application to the AMR criterion triggered by velocity jump does not cause any appreciable increase of memory expense in cosmological numerical simulations, compared to the adoption of the standard AMR criterion. Interestingly enough, although the volume occupied by cells at the highest AMR level is similar in both runs (i.e. $\sim 55$ per cent of the AMR volume of side $7.5 \mathrm{Mpc}$ ), we measure a slightly larger number of refined cells in the d128 run ( $\sim 1-2$ per cent) at all AMR level. Differences in the distributions are consistent with the effect driven by the differences in the thermodynamics of the gas simulated with the two approaches. Indeed, the v128-3 run has a larger amount of turbulent energy (Sect. 4.2) inside the simulated galaxy cluster and this reduces the innermost gas density compared with that of d128; this balances the larger number of cells triggered according to their velocity jump. 

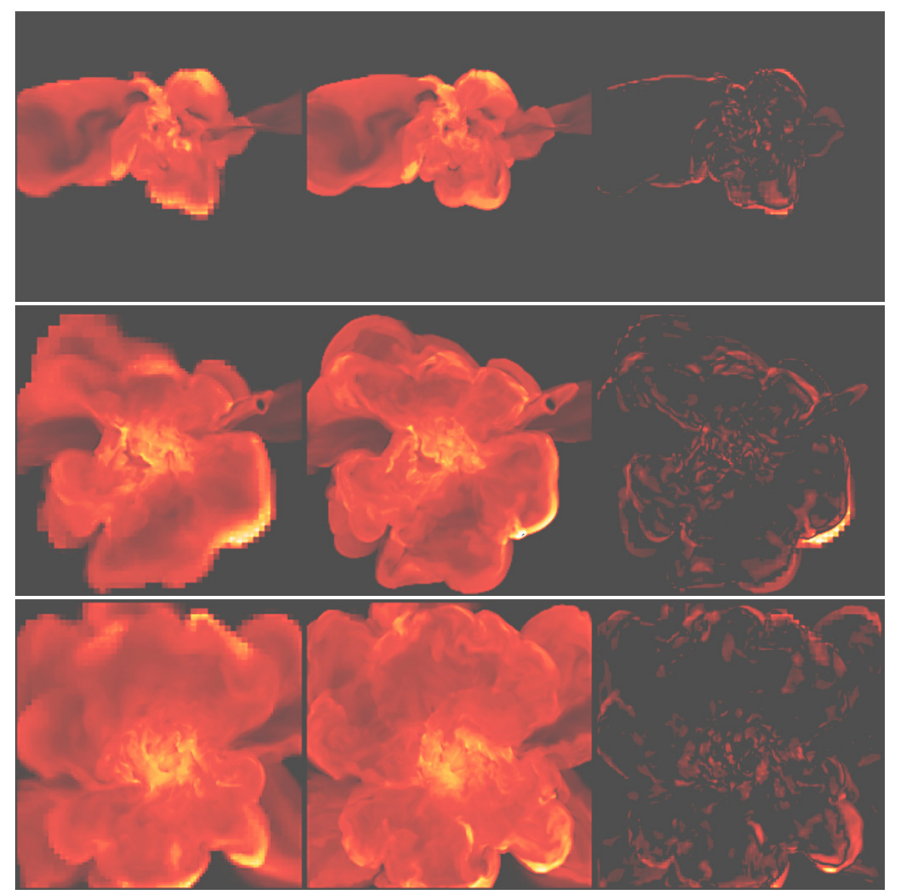

Fig. 3. Temperature maps for a central slice in the simulated AMR, at for different redshifts $(z=1.0, z=0.6$ and $z=0.2)$ by using the standard AMR criterion (d128 run, left panels), the new AMR criterion (v128-3 center panels); the right panels show the cell by cell difference, as $T_{\text {new }}-T_{\text {standard. }}$ The color table is as in Fig. 2, right panels.

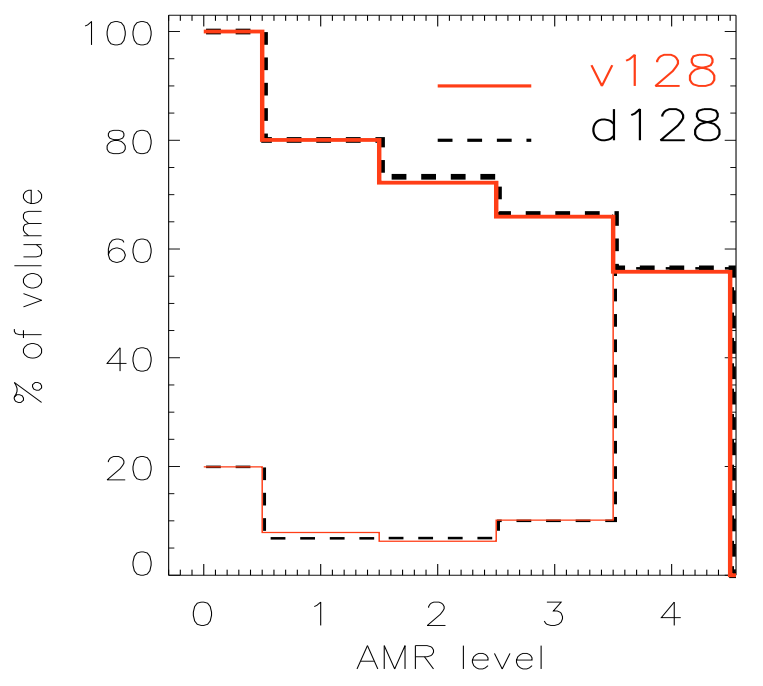

Fig. 4. Ratio of the volume covered by cells at the various AMR levels (normalized to the volume of the AMR region), for runs v128-3 (redsolid) and d128-3 (black-dashed) at $z=0.1$. The thick lines gives the cumulative distribution, while the thin lines show the differential distribution. The root grid level is labeled as " 0 ".

\subsection{Turbulent motions}

In order to characterize turbulent velocity fields in the complex environment of galaxy clusters, it is necessary to extract velocity fluctuations from a complex distribution of velocities. Dolag et al. (2005) proposed that the turbulent gas velocity field can be extracted by removing a "local" mean velocity field, whose value is obtained by interpolating the $3-\mathrm{D}$ gas velocity on large enough scales. Using this approach, it was shown that the bulk of laminar infall motions driven by accreted substructures in smoothed particles hydrodynamics (SPH) simulations develops at scales of the order of $\geq 100-300 \mathrm{kpc}$, which indeed corresponds to the core radii of matter clumps accreted by massive galaxy clusters.

Following a similar approach, here we use the ENZO implementation of the PPM scheme (based on parabolic interpolations on cells) to map the 3-D local mean velocity field, $V_{\mathrm{L}}$, and for each cell we measure the turbulent velocity as $\Delta v=v-V_{\mathrm{L}} ; v$ is the gas velocity at the maximum AMR level, while $V_{\mathrm{L}}$ is measured at a coarser resolution (for the v256-3 and v256-4 runs this is $\Delta=292 \mathrm{kpc}$, while for the other runs we consider the AMR level corresponding to this scale). We notice that this procedure implies a largest possible scale of $\approx 300 \mathrm{kpc}$ for turbulent motions, and therefore in presence of significant turbulent motions on larger scales our procedure would lead to a lower estimate on the total turbulent energy budget. Yet, the influence of our filtering scale in the final estimate of the turbulent energy cannot be larger than a factor $\sim 2$. This simply comes from the comparison of the kinetic energy and turbulent energy profiles reported in the last panel of Fig. 6, and it is consistent with tests previously reported in Dolag et al. (2005) e Vazza et al. (2006). The visual inspection (e.g. Fig. 5) further confirms that most of the velocity structure present in the IGM at scales $>300 \mathrm{kpc}$ is mostly due to laminar infall motions.

In all runs, the total mass of the cluster at the center of the AMR region at $z=0$ is $M \sim 2.1 \times 10^{14} M_{\odot}$, which corresponds to a virial radius of $R_{\text {vir }}=1.4 \mathrm{Mpc}$. Panels in Fig. 5 show the total and turbulent velocity fields for an epoch just after the major merger event, $z=0.6$, for a slice crossing the AMR region. The laminar infall patterns, due to accretion of sub-clumps from filaments (left panel), are almost completely removed by our filtering of the velocity field, and small scale curling motions injected by accreted clumps and by shocks (see also Sect. 4.4) are well highlighted (right panel).

The uppermost panels in Fig. 6 show the gas density profile and the gas entropy profiles of the cluster in all runs. The lower panels in the same Figure show the profiles of thermal, turbulent and kinetic energy, and the ratio between turbulent (or kinetic) energy and the total energy $E_{\text {tot }}$ (kinetic plus thermal) inside a given radius. The turbulent energy, $E_{\text {turb }}$, is measured as $\rho \Delta v^{2} / 2$, the total kinetic energy is $E_{\text {kin }}=\rho v^{2} / 2$ and the thermal energy in the cell is $E_{\text {th }}=(3 / 2) k_{\mathrm{B}} \rho T / \mu m_{\mathrm{p}}$; the velocity field is always corrected for the galaxy cluster center of mass velocity; all profiles refer to $z=0.1$. The standard AMR run (i.e. over-density based refinement, d128) shows the highest central density and the steepest entropy profile, while all runs with velocity/over-density refinement have flatter profiles. This is explained because merger shocks in runs with the velocity/overdensity AMR criterion are simulated with higher accuracy during cluster lifetime, and they can propagate more deeply towards the inner regions of the cluster without being damped by resolution effect. At all radii, the runs with the velocity/over-density refinement show larger energy budget in turbulent motions, with a $E_{\text {turb }} / E_{\text {tot }} \sim 3-4$ percent at $r=0.1 R_{\text {vir }}\left(E_{\text {turb }} / E_{\text {th }} \sim 5\right.$ per cent within the same radius) and $E_{\text {turb }} / E_{\text {tot }} \sim 5-8$ percent inside $R_{\text {vir }}$ ( $E_{\text {turb }} / E_{\text {th }} \sim 10-20$ per cent within the same radius $)$. As expected the adoption of a larger threshold for $\delta$ (v128-10) decreases the budget of turbulent motions in the simulated volume, gradually approaching the results of standard AMR(d128), except for the outermost regions, where strong shocks occur and the threshold $\delta=10$ still triggers refinement. Decreasing $\delta$ (v128-1) increases the turbulent energy budget, yet convergence 

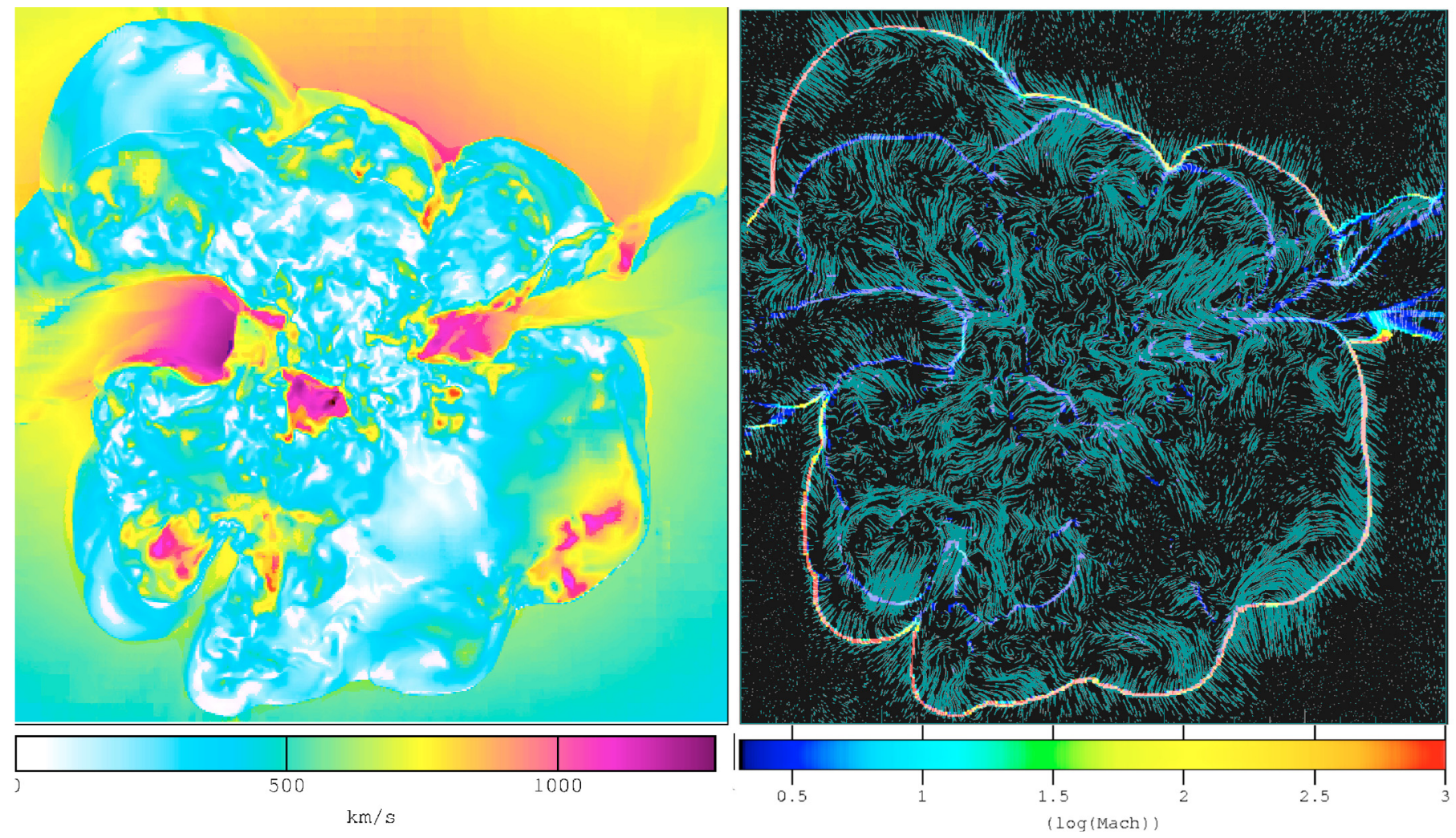

Fig. 5. Left: modulus of total gas velocity in a slice of side $7.5 \mathrm{Mpc}$ and depth $18 \mathrm{kpc}$, for the v256-4 run at $z=0.6$. Right: map of Mach number (in colors) and turbulent gas velocity field (arrows).

is already reached at $\geq 0.2 R_{\mathrm{vir}}$ for $\delta=3$ (v128-3). The adopting the mesh refinement criterion based on velocity jumps for $z<2$ (v128-z2) produces profiles consistent with those from the run where this criterion is applied since the beginning of the simulation (v128), provided a small difference is found for $r \sim R_{\mathrm{vir}}$.

In the cases where the AMR peak resolution is fixed at $\Delta=36 \mathrm{kpc}$ (v256-3, v128-3, v64-3), the adoption of a larger mass resolution in DM particles causes a significant decrease in the turbulent budget at large radii (the kinetic energy profiles, however, are almost unaffected by that). We find that the reason for this is that in the cluster outskirts, where strong accretion shocks are located, satellites with a too coarse DM mass resolution have a typically smaller gas and DM density concentration, and they are more easily stripped and/or destroyed generating more small scale chaotic motions in the peripheral regions of clusters (see also Sect. 4.4).

The total kinetic energy within $R_{\text {vir }}$ in our simulations is in line with SPH results with reduced artificial viscosity (Vazza et al. 2006) and other AMR results obtained with ENZO (Iapichino \& Niemeyer 2008). However, it is unclear if the observed inner turbulence profile can be reconciled with with SPH findings, where an increase of the ratio between turbulent energy and the total one is observed with decreasing radius, for $r / R_{\text {vir }}<0.1$ (Dolag et al. 2005). On one hand it seems that the progressive increase of the DM mass and force resolution in our simulations causes the same kind of steepening also in our innermost profile, on the other hand the turbulent energy budget remains smaller by a factor $\sim 5-6$ respect to SPH results. Whether or not this is related to the different clusters under observation (and to their dynamical states) or if this is this a more fundamental issue caused by differences between AMR and SPH simulations, is a topic that deserves more accurate investigations in the future.

\subsection{Power spectra and structure functions of the turbulent velocity field}

We characterize the cluster velocity field through it 3D power spectrum, $E(k)$, defined as:

$E(\boldsymbol{k})=\frac{1}{2}|\tilde{\boldsymbol{v}}(\boldsymbol{k})|^{2}$

where $\tilde{\boldsymbol{v}}(\boldsymbol{k})$ is the Fourier transform of the velocity field:

$\tilde{\boldsymbol{v}}(\boldsymbol{k})=\frac{1}{(2 \pi)^{3}} \int_{V} \boldsymbol{v}(\boldsymbol{x}) \mathrm{e}^{-2 \pi \mathrm{i} \cdot \boldsymbol{x}} \mathrm{d}^{3} x$.

$E(k)$ is calculated with standard FFT algorithm (e.g. Federrath et al. 2009, and references therein), and with a zero-padding technique to deal with the non-periodicity of the considered volume. Differently from SPH and standard AMR simulations, the velocity plus density refinement allows to follow the cluster velocity field with high spatial resolution in lower density regions, with important consequences on the capability to describe its spectral properties over a wide range of scales.

The left panel in Fig. 7 shows the 3-D power spectra calculated for all runs at $z=0.1 . E(k)$ is approximately described by a simple power law over more than one order of magnitude in $k$, with a slope not far from a standard Kolmogorov model $\left(E(k) \propto k^{-5 / 3}\right)$. At large scales $(k<4)$ a flattening in the spectrum is observed in all runs, at a wave number roughly corresponding to the virial diameter of the cluster, which likely identifies the outer scale of turbulent motions connected with 

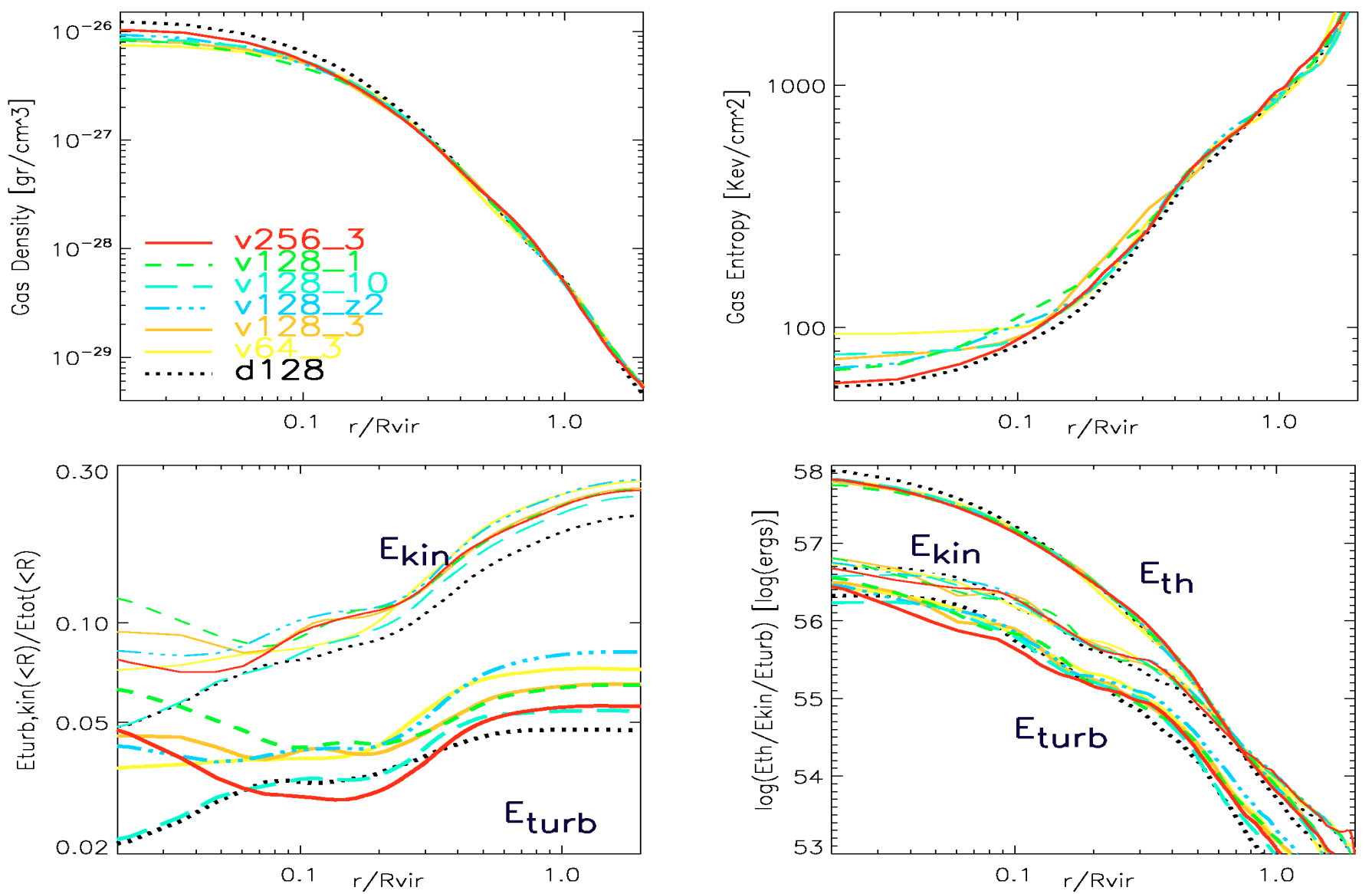

Fig. 6. Gas density profiles (top left), gas entropy profiles (top right), $E_{\text {turb }} / E_{\text {tot }}$ and $E_{\text {kin }} / E_{\text {tot }}$ profiles (lower left) and $E_{\text {turb }}, E_{\text {kin }}$ and $E_{\text {therm }}$ profiles (lower right) for all runs of the paper at $z=0.1$. The color coding is all the same as in the first panel; $E_{\text {turb }}$ refers to gas motions on scales $<300 \mathrm{kpc}$.
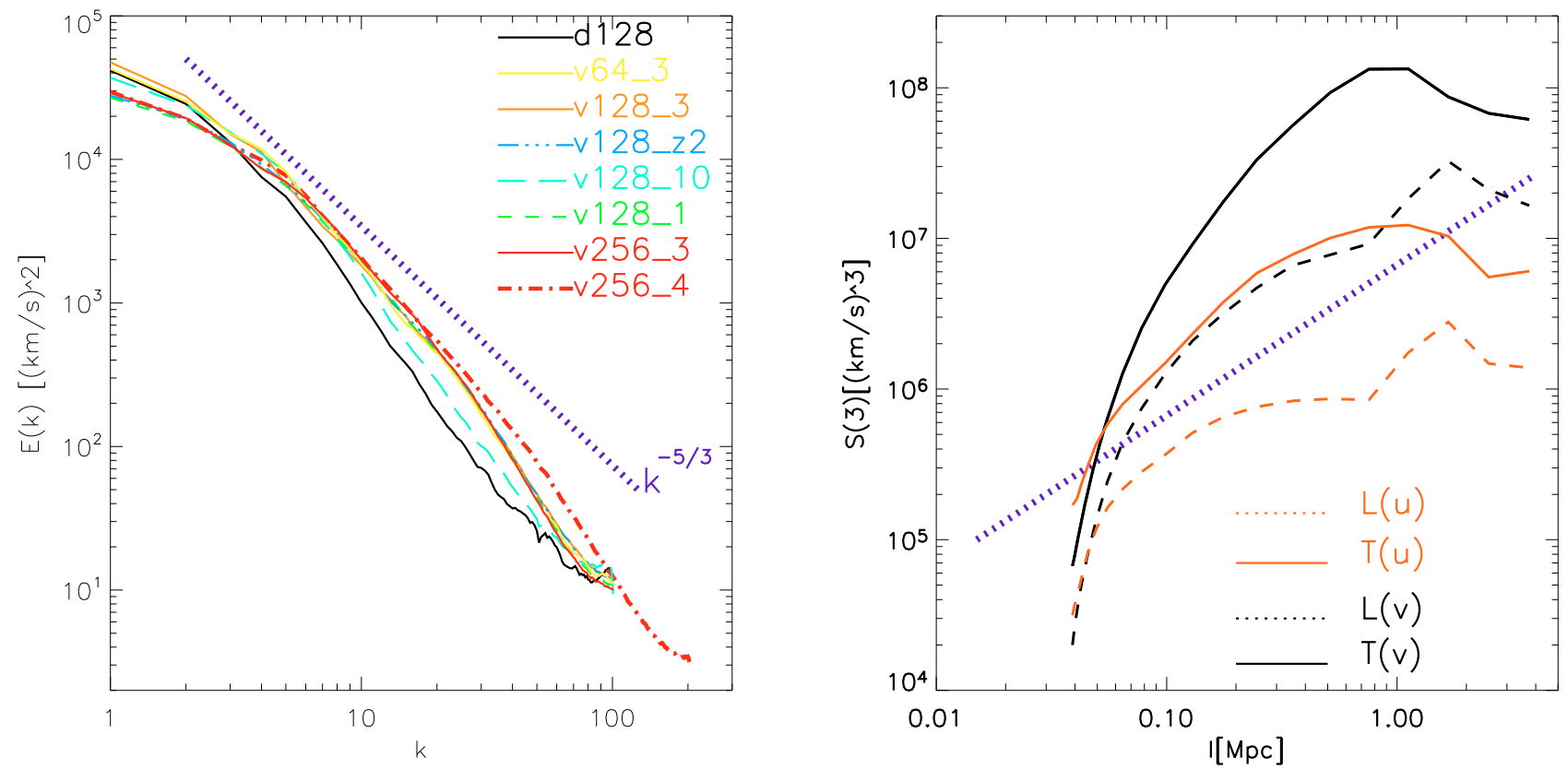

Fig. 7. Left: 3D power spectra for the velocity field of the various run at $z=0.1$. The spectra are shown up to their Nyquist frequency; the purple dashed lines shows the $-5 / 3$ slope to guide the eye. Right: longitudinal and transverse third-order structure functions for velocity field, $v$ (black, and for the density $=$ weighted velocity field, $u \equiv \rho^{1 / 3} v(r e d)$, for a sub volume in the v128-3 run. The purple dashed line shows the expected scaling for the Kolmogorov model. 
accretion processes. We remark that for spatial scales $\leq 32 \Delta$, the slope of the power spectrum is affected by the non-uniform numerical dissipation that PPM adopts to increase resolution in shocks and contact discontinuities (Porter \& Woodward 1994).

As in the case of the turbulent energy budget, the v128-10 run falls in between the standard AMR run and all the other runs with velocity/over-density refinement, while there is almost no difference by adopting $\delta=3$ or $\delta=1$ as threshold; we find no relevant differences if the velocity refinement criterion is adopted at $z<2(\mathrm{v} 128-\mathrm{z} 2)$ or at $z=30$ (v128).

Remarkably due to its larger peak resolution, the v256-4 shows a regular power law for almost two orders of magnitude, thus supporting the picture that the simulated IGM is globally turbulent starting from sub-Mpc scales. This is also further suggested by the right panel in Fig. 7, which shows the third order velocity structure functions for the v128-3 run, calculated as

$S_{\mathrm{p}}(l)=\left\langle|\boldsymbol{v}(\boldsymbol{r}+\boldsymbol{l})-\boldsymbol{v}(\boldsymbol{r})|^{3}\right\rangle$.

Shown are the transverse $(\boldsymbol{v} \perp \boldsymbol{l})$ and longitudinal $(\boldsymbol{v} \| \boldsymbol{l})$ structure functions extracted from a random sub-sample of $\sim 10^{5}$ cells in the simulated volume. For completeness, structure functions are also calculated for the density-weighted velocity, $\boldsymbol{u} \equiv \rho^{1 / 3} \boldsymbol{v}$, which was introduced by Kritsuk et al. (2007) to study scaling relations for simulated supersonic turbulence. All signals show a peak at $\sim$ Mpc scales, thus implying that the maximum outer scale that drives turbulence is of the order of $R_{\mathrm{vir}}$.

\subsection{Shock properties}

Shocks in large scale structures have been investigated in a number of semi-analytical (Gabici \& Blasi 2003; Berrington \& Dermer 2003; Keshet et al. 2003) and numerical works (Miniati et al. 2001; Ryu et al. 2003; Pfrommer et al. 2007; Skillman et al. 2008; Vazza et al.2008; Molnar et al. 2009). Observationally, merger shocks have been detected only in a few in a few nearby X-ray bright galaxy clusters (Markevitch et al. 2005; Markevitch 2006; Solovyeva et al. 2008), and may be possibly associated with single or double radio relics discovered in a number of galaxy clusters (e.g. Roettgering et al. 1997; Markevitch et al. 2005; Bagchi et al. 2006; Bonafede et al. 2009; Giacintucci et al. 2008).

The application of the AMR approach described in this paper to galaxy allows to follow with high resolution the onset and the evolution of shock waves in the IGM of simulated galaxy cluster within $\sim 2 R_{\text {vir }}$ from the clusters center, and to explore the connection between shocks and turbulence in large scale structures.

In Fig. 8 we present standard 1-D shock-tube tests for a weak $(M \approx 1.5)$ and for a strong $(M \gg 10)$ shock, where we compare the application of the AMR criterion based on velocity jumps (with $\delta=3$ ), the application of the density jump criterion (with $\delta_{\rho}=2$ ), and a simulation with constant spatial resolution fixed at the maximum resolution level of the AMR runs. In both tests, the run with AMR based on velocity jumps well matches the results of the fixed resolution run at the position of the traveling shock waves, and as result the correct shock jump conditions can be basically measured across 3 cells, in both cases (e.g. Tasker et al. 2009). On the other hand the AMR based on over density smears the weak shocks across a larger distance, since the small density jumps associated with $M \approx 1.5$ is not large enough to trigger any mesh refinement; however in the case of the $M \gg 10$ shock the gas compression is large enough to trigger two level of refinement also in the AMR method based on over density.
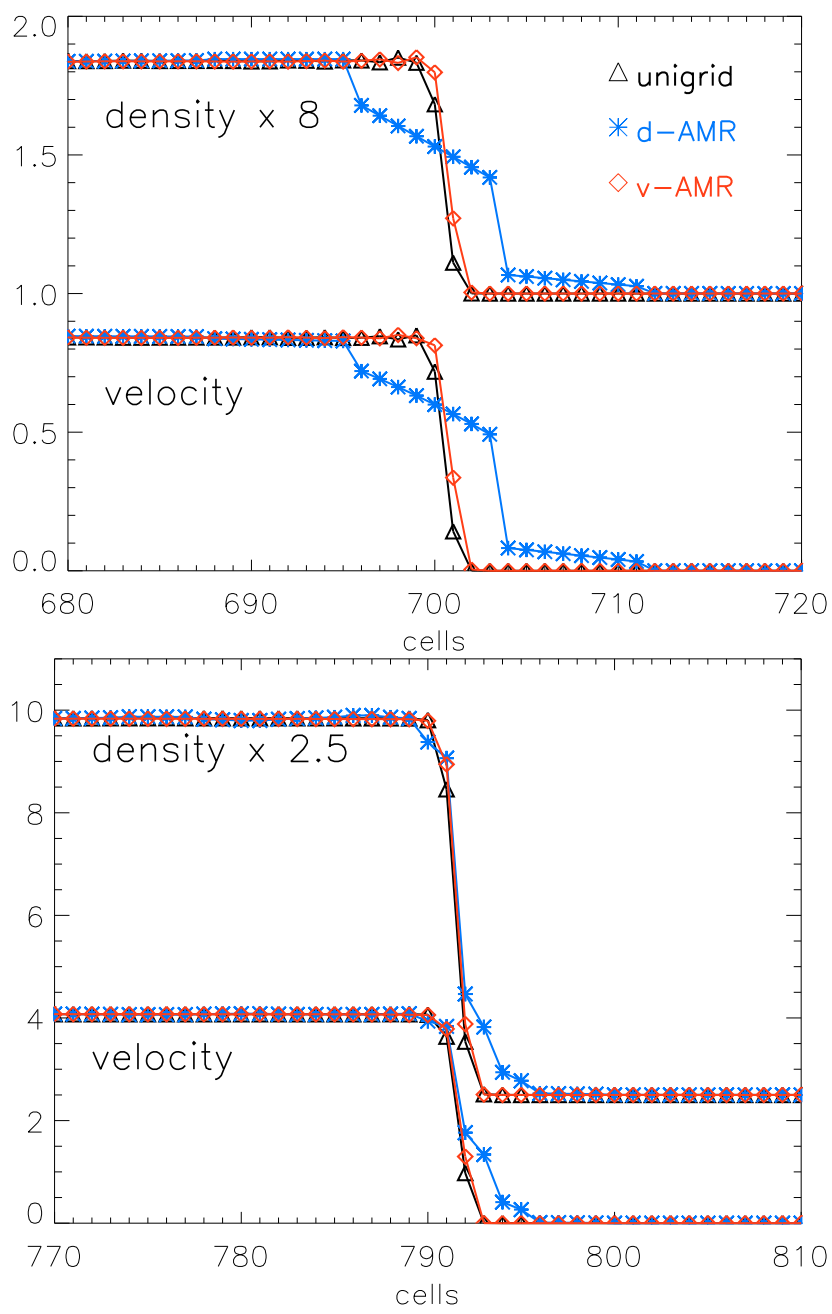

Fig. 8. Top panel: gas density and gas velocity for a 1-D shock tube test, at the position of a $M \approx 1.5$ shock wave. Bottom panel: gas density and gas velocity for a 1-D shock tube test, at the position of a $M \gg 10$ shock wave. In both cases results are shown for a run with $N=1024$ cells with uniform resolution (unigrid), and for two AMR runs with root grid $N=128$ and 3 additional level of refinement, triggered by density jump $(d-A M R)$ and by velocity jump $(v-A M R)$. The values of gas densities in both cases have been rescaled by an arbitrary value to avoid overlapping with the other lines.

Shocks in the our cosmological simulations are identified by means of the procedure presented in Vazza et al. (2009). The algorithm works in the following steps:

- we consider candidate shocked cells those with $\nabla \cdot v<0$ (calculated as 3-dimensional velocity divergence to avoid confusion with spurious 1-dimensional compressions that may happen in very rarefied environments);

- since shocks in the simulation are typically spread over a few cells, we define the shock center with the position of the cell in the shocked region with the minimum divergence;

- we scan the three Cartesian axes with a one-dimensional procedure measuring the velocity jump, $\Delta v_{x, y, z}$, between 3 cells across the shock center;

- the Mach number of the shock is obtained by inverting

$\Delta v=\frac{3}{4} c_{\mathrm{s}} \frac{1-M^{2}}{M^{2}}$, 


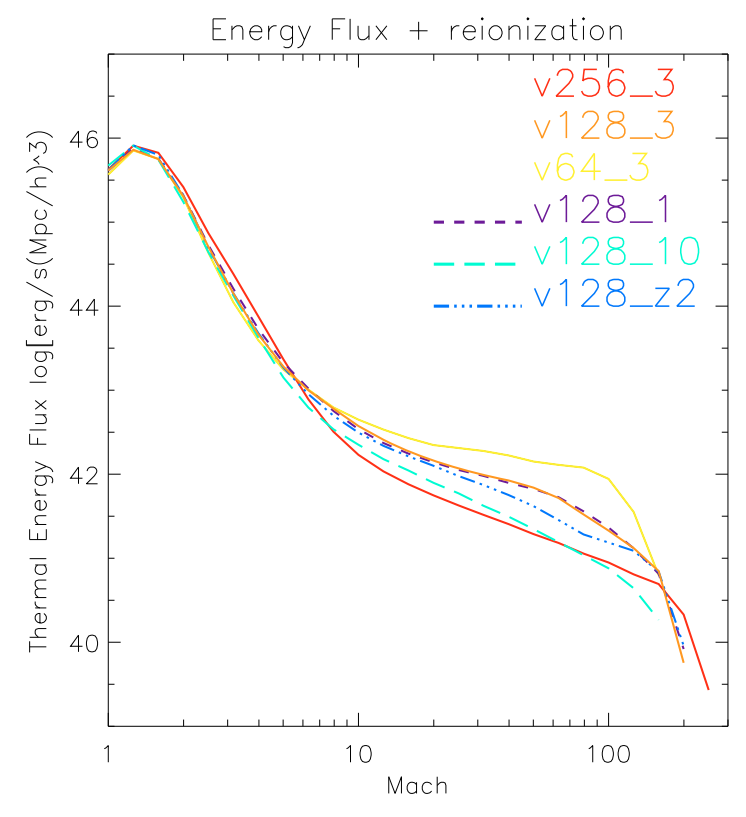

(where $\rho$ is the gas density and $\rho_{0}=10^{-32} \mathrm{~g} \mathrm{~cm}^{-3}$ ) which is found to mimic the integrated effect of the Haardt \& Madau (1996) run-time re-ionization scheme adopted in ENZO with sufficient accuracy.

The Right panel in Fig. 5 shows a map of reconstructed Mach number with this method, for a slice of $18 \mathrm{kpc}$ crossing the AMR region of run v256-4 (with overlaid streamlines of the turbulent velocity field).

In the case that AMR is forced to increase the spatial resolution also around shocks, as our simulations with the novel AMR criterion, the Vazza et al. (2009) shock detecting scheme can be straightforwardly applied to simulated data at the highest available AMR level (therefore excluding our d128 run). We thus analyze shock statistics in all runs employing the over density/velocity AMR criterion at the resolution of $\Delta=36 \mathrm{kpc}$.

The thermal energy flux across shocks is customary evaluated as:

$f_{\mathrm{th}}=\delta_{\mathrm{M}}(M) \cdot \rho M^{3} v_{\mathrm{s}}^{3} / 2$,

where $\rho$ is the pre-shock density and $\delta_{\mathrm{M}}(M)$ is a monotonically increasing function of $M$ (e.g. Ryu et al. 2003).

Figure 9 (top panel) shows the distribution of thermal energy flux at shocks within the AMR region. The distribution of weaker (mostly internal) shocks peaks at $M \sim 1.5$. Overall, the distributions are very steep and consistent with those reported in Vazza et al. (2009). Compared to Pfrommer et al. (2007), who studied shock energetics with high resolution GADGET2 simulations, we find significantly steeper energy flux distributions in all our runs, $\alpha_{\text {th }} \approx-3.5$ (with $f_{\text {th }}(M) M \propto M^{\alpha_{\text {th }}}$ ) for $M<10$, compared to $\alpha_{\text {th }} \approx-2$ within the same range of Mach number in Pfrommer et al. (2007). The distributions of the various re-simulations show relevant differences only for shocks with $M>10$, where two clear trends can be found:

- for a fixed DM mass resolution, the adoption of $\delta=10$ (v128-10)leads to a significant reduction of the thermal energy flux at strong shocks compared to the other runs;

- for a given AMR criterion based on over density/velocity jumps, increasing the DM mass resolution leads to a significant decrease in the thermal energy flux processed at strong shocks and to a progressive steepening of the thermal energy flux distribution.

Fig. 9. Top: distribution of thermal energy flux at shocks within the AMR region of all simulated runs with the over density/velocity AMR criterion, at the grid resolution of $\Delta=36 \mathrm{kpc}$. Bottom: radial profile of the energy-flux weighted average Mach number at $z=0.1$, for all runs with the over density/velocity AMR criterion.

where $c_{\mathrm{s}}$ is the sound speed in the pre-shock region (the cell with the minimum temperature);

- we finally reconstruct the 3-D Mach number in shocked cells as $M=\left(M_{x}^{2}+M_{y}^{2}+M_{z}^{2}\right)^{1 / 2}$, that would minimizes projection effects in the case of diagonal shocks.

In order to have a realistic value of the shock Mach number at accretion shocks, we apply the post-processing re-ionization scheme in Vazza et al. (2009), by increasing the gas temperature inside cells according to:

$T_{\min }(K)=450\left(\frac{\rho}{\rho_{0}}\right)^{0.60}$,

Overall we conclude that, once that the velocity jump AMR criterion is adopted, the largest amount of difference in the spatial and energy distribution of shocks is caused by the DM mass resolution.

In order to highlight the reason for this finding, we show in Fig. 10 the maps of projected Dark Matter density, gas temperature, mean Mach number and thermal energy flux for a slice of depth $=100 \mathrm{kpc}$ for runs v256-3, v128-3 and v64-3. The increase in the number of accreted DM clumps in simulations with higher DM mass resolution is found to generate a more complex temperature distribution, which follows the pattern of matter infall on the cluster. On the other hand when the DM mass resolution is coarse, outer shocks are found to be more regular in shape, and they are characterized by sharper jumps. The decrease of DM mass resolution implies that the cluster becomes more spherically symmetric due to the lack of substructure, thus our findings qualitatively support those of Molnar et al. (2009), which shows that the importance of pressure jumps due to accretion shocks in simulated clusters is reduced by a factor 5-10 compared to predictions based on spherical models (Kocsis et al. 2005). 

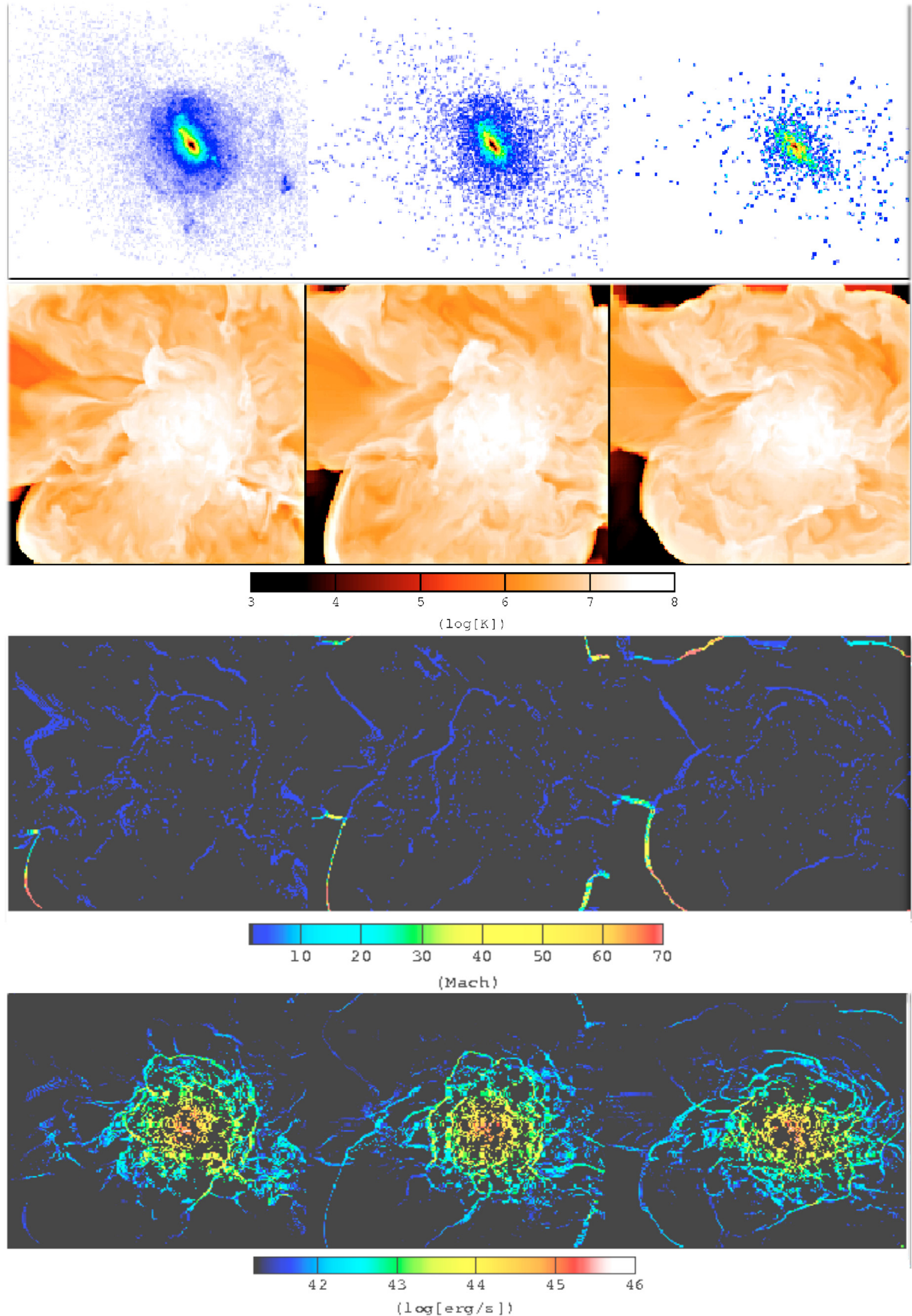

Fig. 10. From top to bottom: a) projected Dark Matter density maps; b) projected volume weighted temperature maps; c) projected thermal energy flux-weighted maps of Mach number; d) projected maps of thermal energy flux at shocks. The left column refers to run d256-3, the center one to d128-3 and the right one to v64-3. Each image has side $7 \mathrm{Mpc}$ and a LOS depth of $100 \mathrm{kpc}$. 

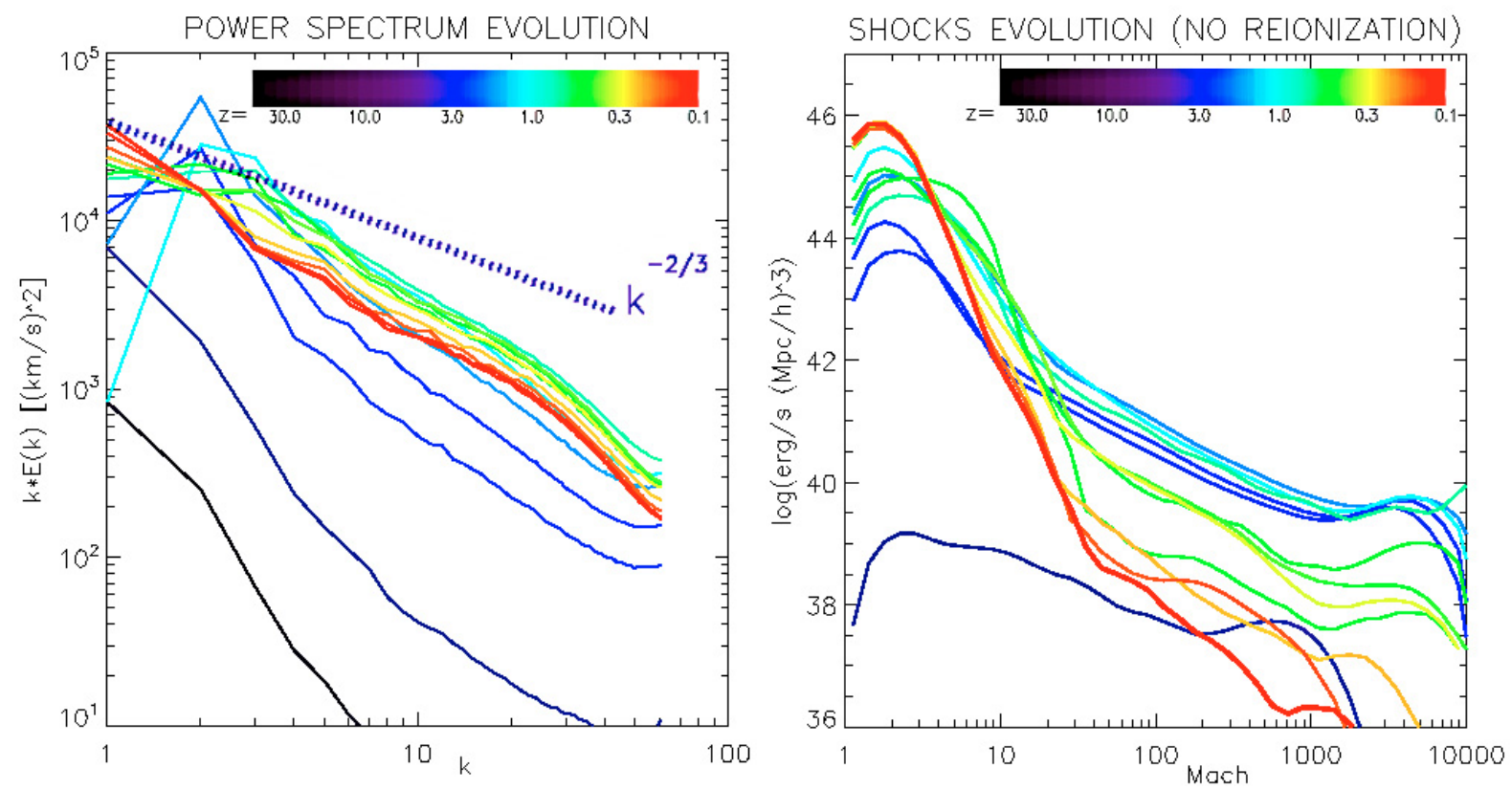

Fig. 11. Left: time evolution from of the $k E(k)$ for a sub-volume of side $3.5 \mathrm{Mpc}$ in the v256-3 run. The additional dashed line shows the slopes for the Kolmogorov model. Right: time evolution of the thermal energy flux at shocks for the same volume. The color coding for the liens is shown in the color bar.

Figure 9 (bottom panel) shows the radial profile of the energy-flux weighted average Mach number for all runs with the over density/velocity refinement. All runs produce consistent profiles up to $R_{\mathrm{vir}}$, with $\langle M\rangle \approx 1.5$. As seen above, differences are larger at accretion shocks outside $R_{\mathrm{vir}}$, and in particular we find that as soon as the DM resolution is increased, the mean strength of shocks at $r \sim 1-2 R_{\mathrm{vir}}$ is reduced by a factor $\sim 2-5$.

\subsection{Time Evolution}

We produced a highly time-resolved study of turbulence and shocks developing in the AMR region of run v256-3. Left panel in Fig. 11 shows the evolution with cosmic time of $k E(k)$ within a sub-volume of $3.5 \mathrm{Mpc}$ centered on the cluster center. The bulk of turbulence injection starts with the onset of the major merger, at $z \sim 1$, and develops at scales in the range $\sim 1-2 \mathrm{Mpc}$. At smaller redshifts, the spectrum gradually approaches the shape reported in Fig. 7.

To better explore the connection between shock waves and turbulence in the merger event, we show in Fig. 11 (right panel) the evolution of the thermal energy flux through shocks for the same sub-volume considered in the Left panel. The energy flux is calculated with the same procedure as in Sect. 4.4, but in this case no treatment of re-ionization is considered, and therefore accretion shocks are stronger than those measured in Fig. 9, because of the unrealistically low value of gas temperature outside the galaxy cluster at evolved redshifts.

A bump of thermal energy flux at strong merger shocks is measured at approximately at the same epoch when the bulk of large scale kinetic energy is injected in the IGM. Soon after virialization occurs, extremely strong shocks become rarer and the shocks energy distribution approaches the distribution of Left panel in Fig. 10 (provided that the considered volume is smaller, and that re-ionization is not modeled here).

\section{Conclusions}

A very simple implementation of a new refinement criterion in ENZO simulations allows to follow shocks and turbulent motions with unprecedented detail up to large distances from cluster centers. This refinement criterion is successful in catching the bulk of turbulent motions developed in the IGM by cluster formation processes, allows us to measure velocity power spectra across two orders of magnitude in spatial scales, and to follow shocks statistics and evolution over time in great detail.

Compared to the standard grid refinement criterion, we find that the extra refinement on velocity jumps causes no significant extra expense of memory storage, and that by construction it readily allows to use accurate shock detecting scheme at the largest available resolution in these simulations. In all the analyzed runs, the simulated IGM is found to host turbulent motions (on scales $<300 \mathrm{kpc}$ ) accounting for a $\sim 5-25$ per cent of the gas thermal energy within $R_{\text {vir }}$. Compared to refinement based on over-density only, the new criterion shows lower inner gas density, flatter entropy profiles, significantly larger turbulence budget at all radii and a larger thermal energy budget processed at accretion shocks. This is due to the sharper representation of shock waves and turbulent motions, and highlights the importance of highly resolving these phenomena in discussing accretion processes in the IGM of galaxy clusters.

When the new over density/velocity AMR criterion is employed, the DM mass resolution is found to play a fundamental role in setting the properties of the turbulence generation and of thermal energy flux at shocks; if DM resolution is increased, infalling matter clumps are less easily destroyed during accretion and they thus inject less turbulence via the ram pressure stripping mechanism. In addition, the complex accretion pattern established in simulations with high DM mass resolution is found to significantly prevent the formation of sharp accretion shocks, compared to runs where the DM mass resolution is coarser. In our simulations we find no relevant differences in the properties of turbulence and shock waves if the extra refinement based on velocity jumps is considered only starting from $z<2$.

Overall, the above results confirm that shocks, turbulence and dark matter clustering are inter-playing key ingredients which modern cosmological numerical simulations need to follow with high order accuracy and high resolution to model the 
thermal (and non thermal) properties of the IGM in a realistic way.

Acknowledgements. F.V. thanks D. Collins, S. Skory and J. Bordner for the support he received while visiting CASS (San Diego), and acknowledges G. Tormen of useful discussions. F.V. thanks M. Nanni and F. Tinarelli for valuable technical support at Radio Astronomy Institute (Bologna). We thanks the anonymous referee for comments which helped us to improve the quality of the paper. We acknowledge partial support through grant ASI-INAF I/088/06/0, and the usage of computational resources under the CINECA-INAF agreement.

\section{References}

Agertz, O., Moore, B., Stadel, J., et al. 2007, MNRAS, 380, 963

Bagchi, J., Durret, F., Lima Neto, G. B., \& Paul, S. 2006, Science, 314, 791

Berger, M. J., \& Colella, P. 1989, JCoPh, 82, 64

Berrington, R. C., \& Dermer, C. D. 2003, ApJ, 594, 709

Bonafede, A., Giovannini, G., Feretti, L., Govoni, F., \& Murgia, M. 2009, A\&A, 494, 429

Brunetti, G., \& Lazarian, A. 2007, MNRAS, 378, 245

Brunetti, G., Setti, G., Feretti, L., \& Giovannini, G. 2001, MNRAS, 320, 365

Brunetti, G., Giacintucci, S., Cassano, R., et al. 2008, Nature, 455, 944

Bryan, G. L., \& Norman, M. L. 1997, ASPC, 123, 363

Bryan, G. L., \& Norman, M. L. 1998, ApJ, 495, 80

Bryan, G. L., Abel, T., \& Norman, M. L., 2001, [arXiv: astro-ph/0112089]

Churazov, E., Forman, W., Jones, C., Sunyaev, R., \& Bohringer, H. 2004, MNRAS, 347, 29

Cassano, R. 2009, [arXiv:0902 .2971]

Churazov, E., Forman, W., Vikhlinin, A., et al. 2008, MNRAS, 388, 1062

Dolag, K., Vazza, F., Brunetti, G., \& Tormen, G. 2005, MNRAS, 364, 753

Enßlin, T. A., \& Vogt, C. 2006, A\&A, 453, 447

Fabian, A. C., Sanders, J. S., Crawford, C. S., \& Ettori, S. 2003, MNRAS, 341, 729

Federrath, C., Klessen, R. S., \& Schmidt, W. 2009, ApJ, 692, 364

Ferrari, C., Govoni, F., Schindler, S., Bykov, A., \& Rephaeli, Y. 2008, SSR

Gabici, S., \& Blasi, P. 2003, ApJ, 583, 695

Giacintucci, S., et al. 2008, A\&A, 486, 347

Govoni, F., Murgia, M., Feretti, L., et al. 2006, A\&A, 460, 425

Graham, J., Fabian, A. C., Sanders, J. S., \& Morris, R. G. 2006, MNRAS, 368, 1369
Guo, F., \& Oh, S. P. 2008, MNRAS, 384, 251

Haardt, F., \& Madau, P. 1996, ApJ, 461, 20

Henry, J. P., Finoguenov, A., \& Briel U. G. 2004, ApJ, 615, 181

Iapichino, L., \& Niemeyer, J. C. 2008, MNRAS, 388, 1089

Inogamov, N. A., \& Sunyaev, R. A. 2003, Astron. Lett., 29, 791

Keshet, U., Waxman E., Loeb, A., Springel, V., \& Hernquist, L. 2003, ApJ, 585, 128

Kocsis, B., Haiman, Z., \& Frei, Z. 2005, ApJ, 623, 632

Kritsuk, A. G., Norman, M. L., \& Padoan, P. 2006, ApJ, 638, L25

Kritsuk, A. G., Norman, M. L., Padoan, P., \& Wagner, R. 2007, ApJ, 665, 416

Markevitch, M. 2006, The X-ray Universe 2005, 604, 723

Markevitch, M., Govoni, F., Brunetti, G., \& Jerius, D. 2005, ApJ, 2627, 733

Miniati, F., Jones, T. W., Kang, H., \& Ryu, D. 2001, ApJ, 562, 233

Mitchell, N. L., McCarthy, I. G., Bower, R. G., Theuns, T., \& Crain, R. A. 2009, MNRAS, 395, 180

Molnar, S. M., Hearn, N., Haiman, Z., et al. 2009, ApJ, 696, 1640

Murgia, M., Govoni, F., Feretti, L., et al. 2004, A\&A, 424, 429

Nagai D., Kravtsov A. V., \& Vikhlinin A. 2007, ApJ, 668, 1

Norman M. L., \& Bryan G. L. 1999, LNP, 530, 106

Norman M. L., Bryan G. L., Harkness R., et al. 2007, [arXiv:0705. 1556]

O'Shea, B. W., Bryan, G., Bordner, J., et al. 2004, [arXiv: astro-ph/0403044]

Ota, N., Fukazawa, Y., Fabian, A. C., et al. 2007, PASJ, 59, 351

Porter, D. H., \& Woodward, P. R. 1994, ApJS, 93, 309

Pfrommer, C., Enßlin, T. A., Springel, V., Jubelgas, M., \& Dolag K. 2007, MNRAS, 378, 385

Ricker, P. M., \& Sarazin, C. L. 2001, ApJ, 561, 621

Röttgering, H. J. A., Wieringa, M. H., Hunstead, R. W., \& Ekers, R. D. 1997, MNRAS, 290, 577

Ryu, D., Kang, H., Hallman, E., \& Jones, T. W. 2003, ApJ, 593, 599

Schekochihin, A. A., Cowley, S. C., Dorland, W., et al. 2009, ApJS, 182, 310

Schuecker, P., Finoguenov, A., Miniati, F., Böhringer, H., \& Briel, U. G. 2004, A\&A, 426, 387

Skillman, S. W., O’Shea, B. W., Hallman, E. J., Burns J. O., \& Norman M. L. 2008, ApJ, 689, 1063

Solovyeva, L., Anokhin, S., Feretti, L., et al. 2008, A\&A, 484, 621

Subramanian, K., Shukurov, A., \& Haugen N. E. L. 2006, MNRAS, 366, 1437

Tasker, E. J., Brunino, R., Mitchell, N. L., et al. 2008, MNRAS, 390, 1267

Teyssier, R. 2002, A\&A, 385, 337

Vazza, F., Tormen, G., Cassano, R., Brunetti, G., \& Dolag, K. 2006, MNRAS, 369, L14

Vazza, F., Brunetti, G., \& Gheller, C. 2009, MNRAS, 395, 1333

Woodward, P., \& Colella, P. 1984, JCoPh, 54, 115 